\title{
Production and decay of baryonic resonances in pion induced reactions
}

\author{
Witold Przygoda ${ }^{1, \star}$ \\ for the HADES Collaboration \\ ${ }^{1}$ Smoluchowski Institute of Physics, Jagiellonian University, 30-048 Krakow, Poland
}

\begin{abstract}
Pion induced reactions give unique opportunities for an unambiguous description of baryonic resonances and their coupling channels. A systematic energy scan and high precision data, in conjunction with a partial wave analysis, allow for the study of the excitation function of the various contributions. A review of available world data unravels strong need for modern facilities delivering measurements with a pion beam. Recently, HADES collaboration collected data in pion-induced reactions on light $\left({ }^{12} \mathrm{C}\right)$ and heavy $\left({ }^{74} \mathrm{~W}\right)$ nuclei at a beam momentum of $1.7 \mathrm{GeV} / \mathrm{c}$ dedicated to strangeness production. It was followed by a systematic scan at four different pion beam momenta $(0.656,0.69$, 0.748 and $0.8 \mathrm{GeV} / \mathrm{c}$ ) in $\pi^{-}-p$ reaction in order to tackle the role of $N(1520)$ resonance in conjunction with the intermediate $\rho$ production. First results on exclusive channels with one pion $\left(\pi^{-} p\right)$ and two pions $\left(n \pi^{+} \pi^{-}, p \pi^{-} \pi^{0}\right)$ in the final state are discussed.
\end{abstract}

\section{Probing baryon resonances}

One of the key issues in the research of nature over the years was the investigation of baryon resonances, i.e. short-lived excited states of nucleons. From experimental point of view a resonance manifests as a peak located around a certain energy in differential cross section. Its composite nature is probed in scattering experiments and is characterized not only by the complex pole position of the scattering amplitude but also by the couplings to the various channels and hence decay branching ratios. Particularly the important region of $1-2 \mathrm{GeV}$ is occupied by light-flavoured baryons. Such baryons are built out of three quarks $(u, d, s)$ with possible spin either $1 / 2$ or $3 / 2$. Accounting for orbital motion, a supermutiplet $\mathrm{SU}(6) \times \mathrm{O}(3)$ defines all combinations resulting in 434 possible resonances. It highly exceeds the number of 112 identified resonances (Particle Data Group [1]) many of which do not have well determined parameters. The resonance properties were tackled by many models, including quark models (for a review see [2] Sec. 6.1), Dyson-Schwinger approach [3] and last but not least, lattice QCD calculations [4]. Those models are still confronting the fundamental question of "missing resonances". A possible explanation (as suggested in [5]) lies in the fact that missing states can couple weakly to the $N \pi$ channel, which is a predominant source of knowledge about baryon properties. Additional difficulty stems from the fact that resonance spectral functions are usually quite broad and various resonances overlap. This challenge triggered great experimental effort resulting in collection of high precision and quality data in meson photo- and electroproduction [6]. In contrary

^e-mail: witold.przygoda@uj.edu.pl 
the world data from pion-induced reactions are often outdated and of poor quality. This discrepancy calls for new facilities (pion beam factories) to provide the missing data, expressed by many scientists in "Physics opportunities with meson beams" [7]. Considering the large number of resonant and non-resonant mechanisms involved, the combined analysis of various exit channels (coupled channels models, see i.e. [8]) with the partial wave analysis approach is mandatory.

\section{World pion-beam data}

Presently, all information on the $N^{*}$ and $\Delta$ resonances listed in the PDG [1] originate from the partial wave analysis of $\pi N$ total, elastic and charge exchange-scattering data performed by three major groups. Two of them (Karlsruhe-Helsinki [9] and Carnegie-Mellon-Berkeley [10]) were using only data from experiments conducted before 1980. The available statistics was 10 kevents for each of the $\pi^{ \pm} p \rightarrow \pi^{ \pm} p$ reaction, 1.5 kevents for $\pi^{-} p \rightarrow \pi^{0} n$, with only $17 \%$ of polarized data. The George Washington University (SAID) is the only group providing the partial wave analysis for the $\pi N$ elastic scattering data. They use also the updated database (13 kevents of each of $\pi^{ \pm} p \rightarrow \pi^{ \pm} p, 3$ kevents of $\pi^{-} p \rightarrow \pi^{0} n$ and 250 events of $\pi^{-} p \rightarrow \eta n ; 25 \%$ of polarized data) [11] and continuously include the new available data sets. For example, $\pi^{ \pm} p$ elastic scattering by EPECUR [12], added to the SAID database, demonstrates how big improvements in statistics are possible with the modern experiments and, in fact, demanded for the low energy data in order to construct the unbiased partial wave amplitudes. Another very important channel is $\pi \pi N$, since many well established resonances couple to it. The most extensive study in the resonance energy region $1.32-1.93 \mathrm{GeV}$ was made by Manley et al. [13] (with an update [14]) within the isobar approximation. The analysis relied on 241214 bubble chamber events, they were divided into 22 energy bins and the simultaneous fit to 2-pion channels (no $\pi^{-} p \rightarrow \pi^{0} \pi^{0} n$ channel) was performed. The single energy solution was extracted for each energy bin and every isobar channel, delivering knowledge on $N^{*}$ coupling to $\rho N, \Delta \pi$ and $\sigma N$ channels. This complete work had great impact on resonance parametrizations used in various transport models (GiBUU [15, 16] and UrQMD [17]). Since then new experimental data became available in the $\pi^{-} p \rightarrow \pi^{0} \pi^{0} n$ channel from the Crystal Ball collaboration (349611 events at the energy range $W=1213-1527 \mathrm{GeV}[18])$ and in the $\pi^{-} p \rightarrow \pi^{+} \pi^{-} n$ channel from the CHAOS collaboration (20 kevents, low energies, $W=1257-1302 \mathrm{GeV}$, [19]) and from ITEP (40 kevents, high energy $W=2060 \mathrm{GeV}$ [20]). The database for $\pi^{+} \pi^{-}$in the energy range $W=1.3-2.0 \mathrm{GeV}$ remains scarce and often lack differential distributions. More generally, there is a strong need for detailed new measurements in all charged channels.

\section{HADES spectrometer and pion beam facility}

The High-Acceptance Di-Electron Spectrometer (HADES) [21] is installed at GSI Darmstadt on SIS18. It provides high acceptance, good charged particle identification $(p / K / \pi / e)$ and good mass resolution of $2-3 \%$ for $e^{+} e^{-}$pairs in the light vector meson mass range. It allows to study both hadron and rare dilepton production in $N+N, p+A, A+A$ in the beam energy range of a few $\mathrm{AGeV}$. Recently, also pion-induced collisions on proton and nuclei delivered high statistics data sets with $\pi^{+} \pi^{-}$and first world measurements of $e^{+} e^{-}$. The idea of a pion beam facility at SIS18 dates back to the beginning of the HADES project (ealy 1990s). The first commissioning tests took place in $1999[22,23]$. A primary ${ }^{14} \mathrm{~N}$ beam is provided by the SIS18 synchrotron with an intensity of $0.8-1.0 \times 10^{11}$ ions/spill on beryllium $\left({ }^{9} \mathrm{Be}\right)$ target, where the secondary pion beam is generated. Pions are transported within the beam line, equipped with 9 quadrupole and 2 dipole magnets, to the HADES target located 33 meters downstream from the production point (Fig. 1). The maximum pion 


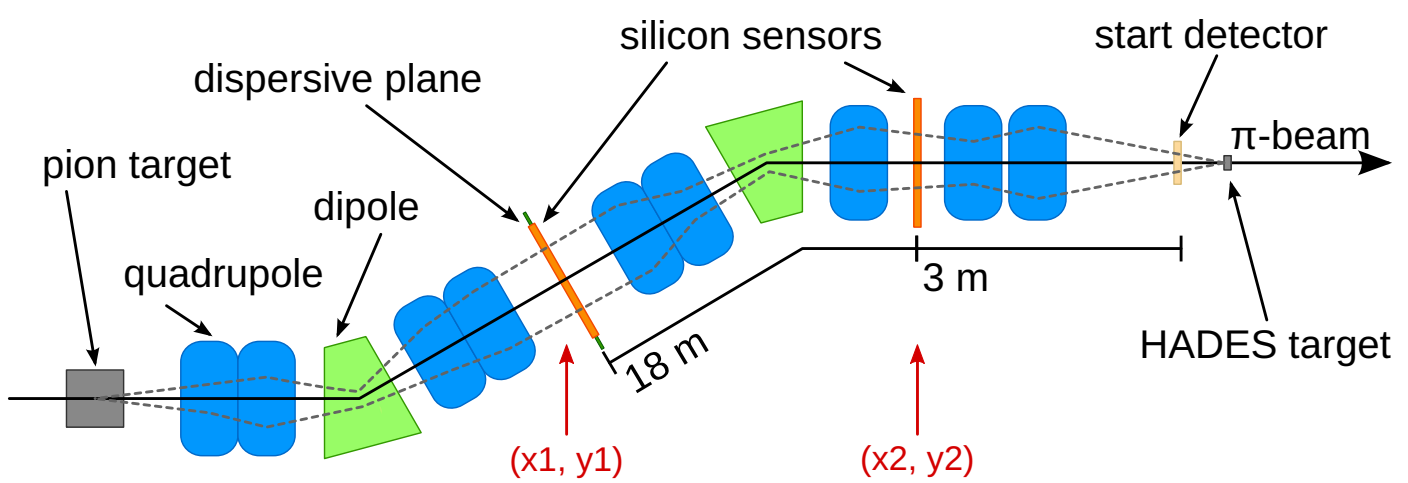

Figure 1. Pion beam line from the production point (pion target) to the HADES target position. Quadrupole and dipole magnets guide the pions (the dashed line) through the in-beam tracking detectors (indicated by the arrows) [26].

intensity of $10^{6}$ pions/spill was achieved at a momentum $p=1.0 \mathrm{GeV} / \mathrm{c}$ and decreased by a factor 2 for the momenta $p=0.7 \mathrm{GeV} / \mathrm{c}$ or $1.3 \mathrm{GeV} / \mathrm{c}$. The dedicated tracking system CERBEROS [26] allows for a reconstruction of the momentum of each pion beam particle with a resolution of $0.3 \%$ (for details, see [24]).

\section{Pion beam results}

The pion beam facility has been operated in 2014 for data taking during an effective period of two weeks. The first run was dedicated to strangeness production in pion-induced reactions on light $\left({ }^{12} \mathrm{C}\right)$ and heavy $\left({ }^{74} \mathrm{~W}\right)$ nuclei at a beam momentum of $1.7 \mathrm{GeV} / \mathrm{c}$. The goal of the second run was to measure the excitation function of two-pion production in the $\pi^{-}-p$ reaction around the pole of the $N(1520) D_{13}$ resonance. Data at four different pion beam momenta $(656,690,748$ and $800 \mathrm{MeV} / \mathrm{c})$ were collected with the largest statistics in the case of the momentum of $690 \mathrm{MeV} / \mathrm{c}$, aimed for dilepton production.

Below, first results of the analysis of three channels (elastic scattering $\pi^{-} p \rightarrow \pi^{-} p$ and two inelastic channels $\pi^{-} p \rightarrow n \pi^{+} \pi^{-}, \pi^{-} p \rightarrow p \pi^{-} \pi^{0}$ ) are presented. In all measurements two targets were used: polyethylene $\left(\mathrm{C}_{2} \mathrm{H}_{4}\right)_{n}$ and carbon $(\mathrm{C})$ respectively, with the aim to subtract events from scattering on carbon and identify pure contribution from scattering on protons. The subtraction procedure involves first establishing a ratio between events collected with the polyethylene target and with the carbon target based on the $\pi^{-} p$ missing mass squared.

\section{$4.1 \pi^{-} p$ elastic scattering}

$\pi^{-} p$ elastic scattering was measured in the same experimental run, serving as the normalization for all other channels. First, events were pre-selected with the help of a cut on coplanar pion and proton reconstructed tracks $\left( \pm 5^{\circ}\right)$ and $\tan \theta_{\pi^{-}} \cdot \tan \theta_{p}>1$ selection. Events originating from scattering on polyethylene target (composed of both carbon and proton atoms in the ratio $1: 2$ ) and carbon target are depicted in Fig. 2 (left) showing $\pi^{-} p$ missing mass squared. The proportion of carbon events (red curve) was scaled such that the left tail, far from the dominating elastic scattering peak on protons, should match the tail from polyethylene events (black curve). This scaling factor is in agreement with the number of all events recorded in the START detector [27] during the measurement with 

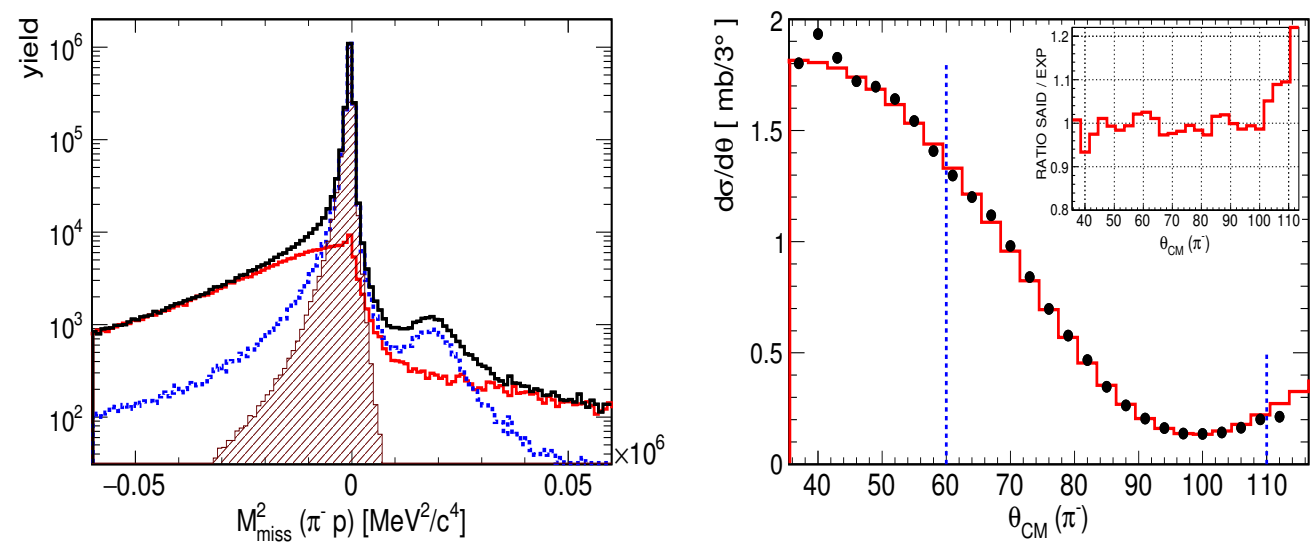

Figure 2. (Color online) Example of $\pi^{-} p \rightarrow \pi^{-} p$ elastic scattering at nominal beam momentum $800 \mathrm{MeV} / \mathrm{c}$ (791.1 MeV/c measured with the pion tracker). Left: uncorrected data, $\pi^{-} p$ missing mass squared with events from $\left(\mathrm{C}_{2} \mathrm{H}_{4}\right)_{n}$ (polyethylene) target (black histogram), $\mathrm{C}$ (carbon) target rescaled (red histogram) and their difference (blue dotted curve) - signal obtained by matching scattering on carbon in polyethylene target with scattering on carbon target on the event by event basis. Finally, elastic scattering selection (dark red dashed area). Right: angular projection of $\pi^{-}$in c.m.s. from $\pi^{-} p$ elastic scattering, corrected for the reconstruction inefficiencies and acceptance (black dots) compared with the distribution from the SAID database [29] within the fiducial volume $60^{\circ}<\theta_{\pi^{-}}^{C M}<110^{\circ}$ (limited by the vertical blue dashed lines). Inset: ratio of SAID data to reconstructed elastic events (red histogram).

the polyethylene target and the carbon target, respectively (after correction for the data acquisition dead time and taking into account different carbon densities of both targets). Scaling factor for the polyethylene and carbon target events, deduced in the elastic scattering channel, holds to all inelastic channels under investigation.

The next step, performed for all investigated channels separately, was matching events of the same kinematic characteristics from polyethylene target and carbon target. Events were grouped into bins of similar missing mass values, then for all combinations among tracks measured with the polyethylene and the carbon target those with the lowest $\chi^{2}$ were paired together. The calculation of the $\chi^{2}$ correlation included a) the momentum and b) polar angle of the measured particles $\left(\pi^{+}\right.$, $\pi^{-}$, proton) and c) invariant mass $\left(\pi^{+} \pi^{-}\right.$and $p \pi^{-}$, respectively). This method delivers pure pionproton reaction candidates. The event by event tagging and rejection of carbon events allows also for the further cut refinement, i.e. elastic scattering selection what is not possible when having also events with the pion scattering on carbon. A few approaches were investigated (i.e. two-dimensional graphical cuts on angle and momentum of $p$ and $\pi^{-}$or condition on the same momentum of particles in the c.m.s.) in order to estimate the systematic error of the elastic scattering identification.

To describe elastic scattering, events were generated in the framework of the PLUTO event generator [25] with the angular parameterization taken from [28]. Then, the full GEANT simulation of the detector response, followed by the same analysis strategy as used for the experimental data, were performed. Simulated elastic scattering events describe the measured data within the HADES acceptance very well and are used for the one-dimensional acceptance correction. The contribution from quasi-elastic scattering from $\mathrm{C}$ (red histogram) has been subtracted based on the missing mass dis- 

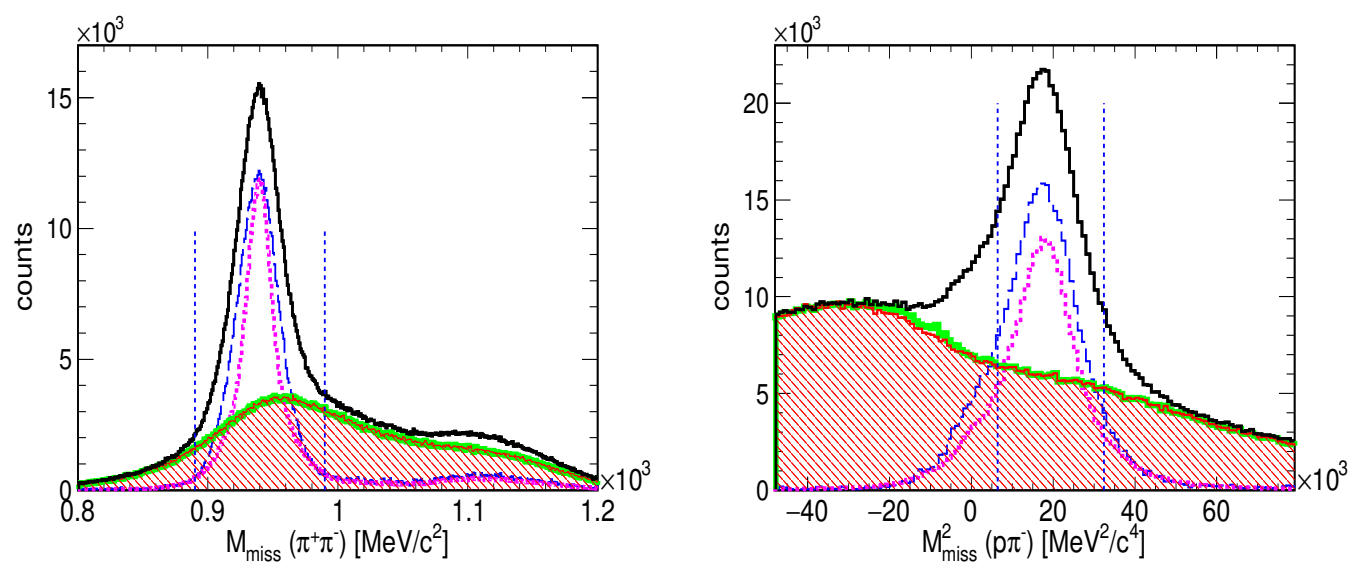

Figure 3. (Color online) Example of missing masses for pion beam scattering at a beam momentum $800 \mathrm{MeV} / \mathrm{c}$. Both figures: uncorrected data, black curve - events from polyethylene target, red curve and red dashed area events from carbon target, green thick curve (behind red) - events from polyethylene target matching events from carbon target, blue dashed curve - signal from scattering on protons from polyethylene target, magenta dotted curve - signal calculated based on information on momentum measured by the pion tracker. The vertical blue dashed lines limit a window ( $\pm 50 \mathrm{MeV} / \mathrm{c}^{2}$ around the neutron missing mass) for selection of events for the partial wave analysis. Left: $\pi^{+} \pi^{-}$missing mass. Right: $p \pi^{-}$missing mass squared.

tributions measured with polyethylene and carbon separately (as described above) and the difference agrees with the line shape expected for $\pi^{-} p$ events obtained from the full Monte-Carlo simulations. The simulations took into account the momentum reconstructed with the help of the pion tracker, with the most probable values of $654.1 \mathrm{MeV} / \mathrm{c}, 683.5 \mathrm{MeV} / \mathrm{c}, 738.9 \mathrm{MeV} / \mathrm{c}$ and $791.1 \mathrm{MeV} / \mathrm{c}$. The corrected data are normalized to the distribution from the SAID database [29] of the known differential $\pi^{-} p$ elastic scattering. It amounts to $3.01 \mathrm{mb}, 3.10 \mathrm{mb}, 3.08 \mathrm{mb}$ and $2.59 \mathrm{mb}$ for the subsequent pion beam momenta in the polar angle range between $60^{\circ}-110^{\circ}$ in c.m.s. The corrected data for the pion beam momentum 791.1 MeV/c (nominal value $800 \mathrm{MeV} / \mathrm{c}$ ) are presented in Fig. 2. The deviation between SAID and experimental values is in the range 2-3\% (root-mean-square error).

\section{$4.2 n \pi^{+} \pi^{-}$and $p \pi^{-} \pi^{0}$ channels}

Identification of two charged pions $\left(\pi^{+} \pi^{-}\right)$or proton and pion $\left(\mathrm{p} \pi^{-}\right)$in the final state allows for the reconstruction of the kinematically complete exclusive channels via cuts in the missing mass distributions around the value of the not detected neutron (Fig. 3 left) or neutral pion (Fig. 3 right). Events from the reactions on proton are deduced after subtraction of the reactions on carbon, as described earlier in this paper. The obtained resolution of the $\pi^{+} \pi^{-}$missing mass peak amounts to $1.5 \%(1 \sigma)$ for a fixed beam momentum or even less, $0.8-1.0 \%$, when using the beam momentum reconstructed in the pion tracker for each event. In both cases the peak position is at the expected value of the neutron mass with an accuracy of $\pm 1 \mathrm{MeV} / \mathrm{c}^{2}$. The resolution of the $p \pi^{-}$missing mass squared is above $40 \%(1 \sigma)$ and above $30 \%$ for the pion tracker momentum used in the reconstruction. The peak position is slightly lower (3-4 MeV/c $\mathrm{c}^{2}$ ) than $135 \mathrm{MeV} / \mathrm{c}^{2}\left(\pi^{0}\right.$ mass) and close (1-2 MeV/c below) in the case of the pion tracker momentum. 

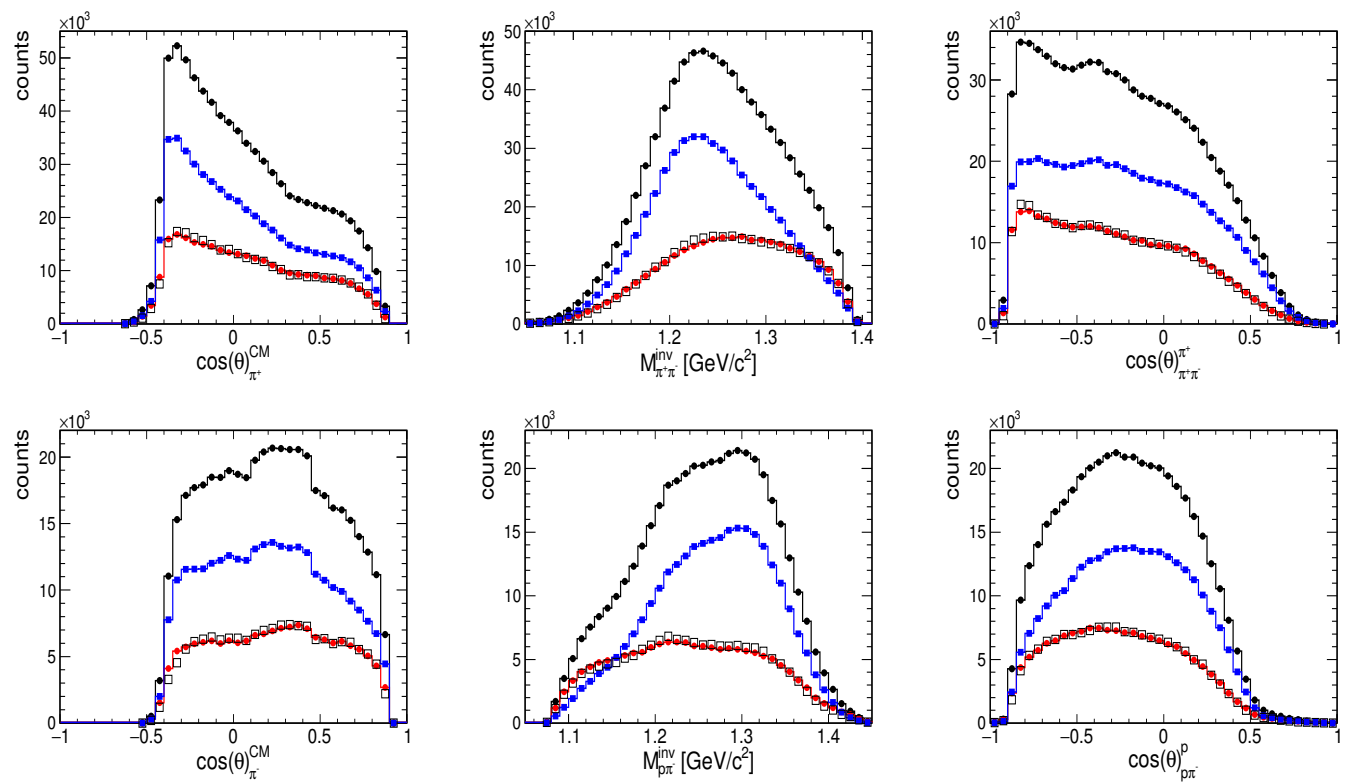

Figure 4. (Color online) Selected projections of $\pi^{-} p$ inelastic scattering at $800 \mathrm{MeV} / \mathrm{c}$ beam momentum in two pion channels. Events from $\left(\mathrm{C}_{2} \mathrm{H}_{4}\right)_{n}$ (polyethylene) target (black histogram), $\mathrm{C}$ (carbon) target (red histogram), events from scattering on protons from polyethylene target (blue histogram), events from polyethylene target matching events from carbon target (black open squares). Upper row: $n \pi^{+} \pi^{-} n$ channel with events from missing mass window (see Fig. 3 left), from left to right, $\pi^{+}$angular distribution in c.m.s., $\pi^{+} \pi^{-}$invariant mass, $\pi^{-}$ angular distribution in Godfried-Jackson (GJ) reference frame of $\pi^{+} \pi^{-}$. Lower row: $p \pi^{-} \pi^{0}$ channel with events from missing mass window (see Fig. 3 right), from left to right, $\pi^{-}$angular distribution in c.m.s., $p \pi^{-}$invariant mass, $p$ angular distribution in Godfried-Jackson (GJ) reference frame of $p \pi^{-}$.

Fig. 4 presents a verification that the selected carbon events from the polyethylene target match events from carbon target not only in the missing mass spectrum (Fig. 3) but also in various projections, i.e. angular distributions and invariant mass. This is the proof that the subtraction of carbon-like events from the polyethylene sample allows for the identification of scattering on protons only. The number of reconstructed events in the $n \pi^{+} \pi^{-}$exclusive channel amounts to more than 400000 (beam momentum $656 \mathrm{MeV} / \mathrm{c}), 7900000(690 \mathrm{MeV} / \mathrm{c}), 815000(748 \mathrm{MeV} / \mathrm{c})$ and $526000(800 \mathrm{MeV} / \mathrm{c})$. The number of reconstructed events in the $p \pi^{-} \pi^{0}$ exclusive channel amounts to more than 122000 (beam momentum $656 \mathrm{MeV} / \mathrm{c}), 3100000$ (690 MeV/c), $490000(748 \mathrm{MeV} / \mathrm{c})$ and $368000(800 \mathrm{MeV} / \mathrm{c})$. Those numbers dramatically improve the world data base of two-pion production in pion-induced experiments in the energy range around the $N(1520)$ resonance. These data will allow for investigation of the branching ratios of this resonance, with a special interest for the $\rho N$ decay. 


\subsection{Analysis strategy}

The two-pion data samples, measured at the four above mentioned pion beam momenta, have been included into the multichannel partial wave analysis developed by the Bonn-Gatchina group [30, 31]. This analysis includes pion induced reactions (including Crystal Ball data [18] with neutral two-pion channel) and a large number of pseudoscalar meson photoproduction data taken with polarized beam and target. The analysis also includes the information about photoproduction of vector mesons. This work is in progress.

In parallel to hadronic channels, the identification of dilepton pairs in the $n e^{+} e^{-}$exclusive channel within the neutron missing mass window and $e^{+} e^{-}$invariant masses above the $\pi^{0}$ mass, in conjunction with the results from the $\pi^{+} \pi^{-}$analysis, will shed more light on the $\rho$ meson production mechanism (see Federico Scozzi contribution [32] to this conference).

\section{Future plans}

The pion beam program will be continued in 2018 once the SIS18 will have been upgraded to serve as an injector for the FAIR facility. In the year 2016 the HADES spectrometer will be extended by a large area electromagnetic calorimeter (ECAL) [33-36], replacing the PreSHOWER detector [37]. The ECAL will enable measurements of real photons emitted from nuclear matter and neutral meson production via their photonic decays (e.g. $\pi^{0} / \eta \rightarrow \gamma \gamma$ ). It will also improve the electron-hadron separation of the spectrometer for the purpose of future experiments of HADES at FAIR in elementary and heavy ion collisions in the energy range of 2-10 AGeV. The reconstruction of neutral mesons is essential also for the complete partial wave analysis of $\pi^{-} N$ reactions.

The HADES Collaboration gratefully acknowledges the support by the grants PTDC/FIS/113339/2009 LIP Coimbra, NCN 2013/10/M/ST2/00042 SIP JUC Cracow, Helmholtz Alliance HA216/EMMI GSI Darmstadt, VH-NG-823, Helmholtz Alliance HA216/EMMI TU Darmstadt, 283286, 05P12CRGHE HZDR Dresden, Helmholtz Alliance HA216/EMMI, HIC for FAIR (LOEWE), GSI F\&E Goethe-University, Frankfurt VHNG-330, BMBF 06MT7180 TU München, Garching BMBF:05P12RGGHM JLU Giessen, Giessen UCY/341123100, University Cyprus, CNRS /IN2P3, IPN Orsay, Orsay MSMT LG 12007, AS CR M100481202, GACR 13-06759S NPI AS CR, Rez EU Contract No. HP3-283286.

\section{References}

[1] K.A. Olive et al. (Particle Data Group), Chin. Phys. C 38, 090001 (2014)

[2] V. Crede and W. Roberts, Rep. Prog. Phys. 76, 076301 (2013)

[3] C.D. Roberts and A.G. Williams, Prog. Part. Nucl. Phys. 33, 477 (1994)

[4] R. Edwards et al., Phys. Rev. D 84, 074508 (2011)

[5] R. Koniuk and N. Isgur, Phys. Rev. Lett. 44, 2720 (1980)

[6] I.G. Aznauryan and V.D. Burkert, Prog. Part. Nucl. Phys. 67, 1 (2012)

[7] W.J. Briscoe et al., Eur. Phys. J. A 51, 129 (2015)

[8] V. Shklyar, H. Lenske and U. Mosel, Phys. Rev. C 93, 045206 (2016)

[9] G. Höhler, Pion-Nucleon Scattering, Landold-Börnstein (1983) vol I/9b2

[10] R.E. Cutkosky et al., Phys. Rev. D 20, 2839 (1979)

[11] R.A. Arndt et al., Phys. Rev. C 74, 045205 (2006)

[12] I.G. Alekseev et al. (EPECUR), Phys. Rev. C 91, 025205 (2015) 
[13] D.M. Manley, R.A. Arndt, Y. Goradia, V.L. Teplitz, Phys. Rev. D 30, 904 (1984)

[14] D.M. Manley and E.M. Saleski, Phys. Rev. D 45, 4002 (1992)

[15] O. Buss et al., Phys. Rept. 512, 1 (2012)

[16] J. Weil, H. van Hees and U. Mosel, Eur. Phys. J. A 48, 111 (2012)

[17] S. A. Bass et al., Prog. Part. Nucl. Phys. 41, 255 (1998)

[18] S. Prakhov et al. (Crystal Ball), Phys. Rev. C 69, 045202 (2004)

[19] M. Kermani et al. (CHAOS), Phys. Rev. C 58, 3431 (1998)

[20] I.G. Alekseev et al., Phys. Atom. Nucl. 61, 174 (1998)

[21] G. Agakishiev et al. (HADES), Eur. Phys. J. A 41, 243 (2009)

[22] R.S. Simon, Prog. Part. Nucl. Phys. 42, 247 (1999)

[23] J. Díaz et al., Nucl. Instrum. Meth. A 478, 511 (2002)

[24] W. Przygoda, JPS Conf. Proc. 10, 010013 (2016)

[25] I. Fröhlich et al., PoS ACAT2007 076 (2007)

[26] R. Lalik et al., 2013 IEEE Nuclear Science Symposium and Medical Imaging Conference (2013), DOI: 10.1109/NSSMIC.2013.6829440

[27] J. Pietraszko, L. Fabbietti, W. Koenig, M. Weber (HADES), Nucl. Instr. Meth. A 618, 121 (2010)

[28] A.D. Brody et al., Phys. Rev. D 3, 2619 (1971)

[29] SAID database: http://gwdac.phys.gwu.edu/

[30] A. Anisovich, E. Klempt, A.V. Sarantsev and U. Thoma, Eur. Phys. J. A 24, 111 (2005)

[31] A.V. Sarantsev, JPS Conf. Proc. 10, 010005 (2016)

[32] F. Scozzi, "Studying $\rho N$ couplings with HADES in pion-induced reactions", MESON 2016

[33] W. Czyzycki et al., arXiv:1109.5550 [nucl-ex]

[34] O. Svoboda et al. (HADES), JINST 9, C05002 (2014)

[35] P. Rodríguez-Ramos et al. (HADES), EPJ Web Conf. 81, 06009 (2014)

[36] O. Svoboda et al., J. Phys. Conf. Ser. 599, 012026 (2015)

[37] A. Balanda et al. (HADES), Nucl. Instrum. Meth. A 531, 445 (2004) 